\title{
Transmission of an Undiagnosed Sarcoma to Recipients of Kidney and Liver Grafts Procured in a Non-Heart Beating Donor
}

\author{
Olivier Detry, ${ }^{1}$ Arnaud De Roover, ${ }^{1}$ Laurence de Leval, ${ }^{3}$ Christian Herens, ${ }^{4}$ \\ Jean Delwaide, ${ }^{2}$ Pierre Honoré, ${ }^{1}$ and Michel Meurisse ${ }^{1}$
}

Transmission of an undiagnosed cancer with solid organ transplantation is a rare but dreadful event. In this paper the authors report the transmission of an undiagnosed sarcoma to recipients of kidney and liver grafts procured in a Maastricht category 3 non-heart beating donor. To the authors' knowledge this case is the first report of such a transmission with a liver graft procured in a non-heart beating donor. The cancer transferal was diagnosed 1 year after transplantation in the recipients of the liver and of one kidney. The liver recipient died from multiple organ failure after a failed attempt of tumor resection. The kidney recipient underwent immunosuppression withdrawal and transplantectomy. Non-heart beating donors should not be particularly at risk for undiagnosed cancer transmission if the procurement is performed according to the same rules of careful inspection of the abdominal and thoracic organs. After diagnosis of donor cancer transmission, kidney recipients should have the graft removed, and immunosuppression should be interrupted. The management of liver graft recipients is very difficult in this setting, and long-term survival was very rarely reported. (Liver Transpl 2005;11:696-699.)

$\mathrm{T}$ ransmission of cancer with solid organ transplantation is a rare but dreadful event. ${ }^{1,2}$ To limit the risk of occurrence of this severe complication, brain dead patients with past history of malignant disease are often excluded from the donor pool, with the relative exception of patients with cerebral nervous system malignant tumors. ${ }^{3,4}$ However, this policy does not eliminate the risk of an undiagnosed donor malignancy, and case reports of undiagnosed cancer transmission have published. ${ }^{5}$

As the transplant community faces an increasing donor organ shortage, the use of marginal

Abbreviation: NHBD, non-heart beating donor.

From the Departments of ${ }^{I}$ Abdominal Surgery and Transplantation, ${ }^{2}$ Hepatogastroenterology, ${ }^{3}$ Pathology, and ${ }^{4}$ Genetics, University of Liège, CHU Sart Tilman, Liège, Belgium.

Address reprint requests to Olivier Detry, Department of Abdominal Surgery and Transplantation, University of Liège, CHU Sart Tilman B35, B-4000 Liège, Belgium. Telephone: 32 43667645; FAX: 32 43667069; E-mail: Oli.Detry@chu.ulg.ac.be

Copyright (c) 2005 by the American Association for the Study of Liver Diseases

Published online in Wiley InterScience (www.interscience.wiley.com). DOI 10.1002/lt.20457 donors, including older donors or non-heart beating donors (NHBDs), is now more widely accepted to partially cover the need of organs. ${ }^{6}$ In this paper the authors report the transmission of an undiagnosed sarcoma to recipients of kidney and liver grafts procured in a Maastricht category 3 NHBD. To the authors' knowledge this case is the first report of such a transmission with a liver graft procured in a NHBD.

\section{Case Report}

\section{Donor}

The donor was a 60-year-old woman without any history of malignant disease. She suffered from deep hypoxia due to a severe acute asthma crisis. She was found in cardiac arrest and circulation was successfully restored after tracheal intubation. She suffered from deep cerebral anoxia. Cortical electro-encephalogram remained isoelectric after 7 days. She was proposed as Maastricht category 3 NHBD, and both kidneys and liver were procured after 5 minutes of cardiac arrest after life support withdrawal in the operative room. No tumor was found during the procurement, which included median laparotomy and sternotomy. No postmortem autopsy was performed. The liver was locally transplanted and the kidneys were allocated to recipients in other centers according to the best matching.

\section{Liver Recipient}

The recipient of the liver was a 53-year-old male patient with hepatitis $\mathrm{C}$ virus cirrhosis and a 3-cm hepatocellular carcinoma. While on the waiting list he underwent chemoembolization and was deemed the best suitable recipient for this NHBD liver. The transplantation procedure and the early postoperative period were uneventful. The tumor was completely necrotic at pathology. The patient was discharged at postoperative day 15 and his evolution was uncomplicated until postoperative month 13, when he presented with cholestasis. Liver ultrasonography and computed tomography showed a large necrotic lesion within the left liver (Fig. 1). Laparotomy confirmed a large tumor that was proven a 


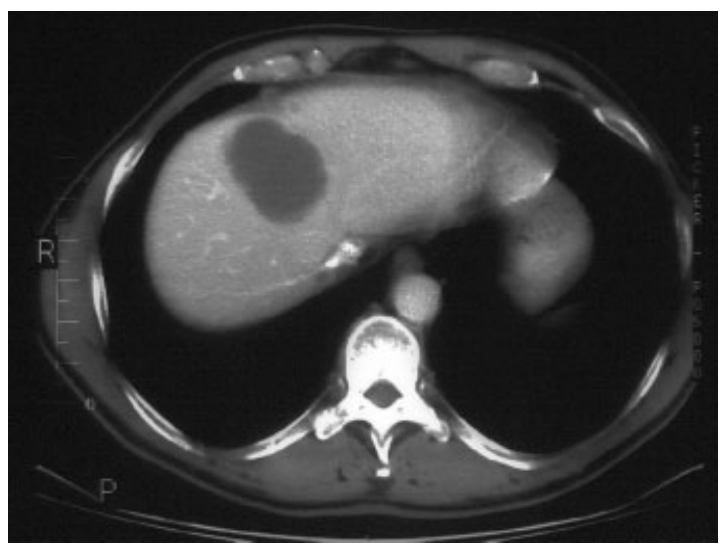

Figure 1. Computed tomography of the liver recipient showing the large necrotic tumor localized in the left liver lobe.

sarcoma at frozen section. R0 resection was impossible, and a palliative hepatico-jejunostomy was performed. The patient died 5 days later from multiorgan failure. Definitive histological sections confirmed a spindle cell sarcoma (Fig. 2). Immunohistochemistry indicated that the cells were diffusely strongly positive for vimentin, negative for cytokeratins, negative for $\mathrm{S} 100$ protein, negative for CD31 and CD34, negative for desmin and CD117, focally faintly positive for smooth muscle actin and focally positive for CD10. Fluorescence in situ hybridization showed an XX pattern in $74.5 \%$ of the nuclei (with polyploidy for the $\mathrm{X}$ chromosome in the tumor cells), establishing the probable donor (female) origin of the sarcoma (Fig. 3).

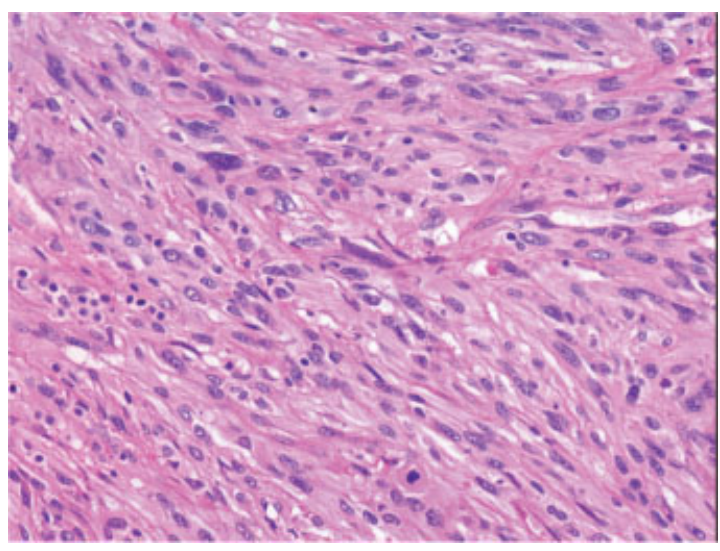

Figure 2. Surgical biopsy of the liver graft tumor showing a spindle cell sarcoma (Hematoxylin $\&$ eosin stain; original magnification $\times 200)$.

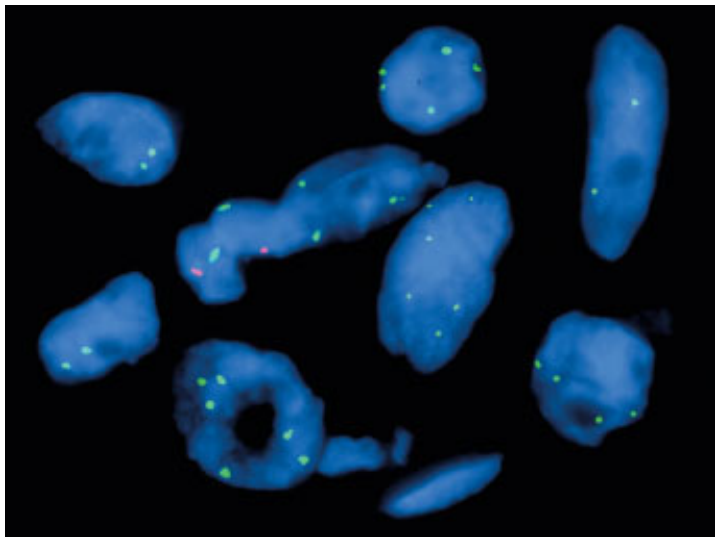

Figure 3. Fluorescence in situ hybridization of the liver graft tumor showing a majority of nuclei with only green signals (chromosome $\mathrm{X}$ ). The chromosome $\mathrm{Y}$ probe (DYZ3) is labeled in red. Note also the presence of polyploid cells for the $\mathrm{X}$ chromosome.

\section{Kidney Recipients}

One kidney was removed 10 days after transplantation because of primary nonfunction. Pathology did not find evidence of tumor, and the recipient did not develop cancer in 18 months of follow-up. The recipient of the second kidney had to undergo transplantectomy 1 year after transplantation for a malignant graft tumor that proved to be a spindle cell sarcoma comparable to the tumor found in the liver recipient.

\section{Discussion}

Transmission of cancer from organ donor to recipient is a sporadic but dreadful complication of solid organ transplantation. Most cases occurred in the pioneering years of organ transplantation, when the risk of cancer transmission was not known. ${ }^{1,2,7}$ Currently, past history of cancer has become a contraindication to organ harvesting, with the relative exception of some tumors with very low risk of dissemination, such as low-grade skin cancers, carcinoma in situ, or primary central nervous system tumors. $3,4,8$ Despite this policy, cases of undiagnosed donor cancer transmission with the graft are still regularly published..$^{5,9.10}$ In this paper, the authors report what they believe is the first case of cancer transmission with grafts harvested in a NHBD in the modern age of organ transplantation. In the 1960s, NHBDs were regularly organ donors, and cases of transmission of cancer with kidney transplants were reported at that time..$^{1,2,7}$ The occurrence of the same tumor in both recipients at nearly the same moment after transplantation, together with the demonstration 
of the female (XX) character of the tumor cells in a male (XY) recipient, proved that this sarcoma was present and undiagnosed in the donor at the time of organ procurement. The primary lesion is not known, and it is probable that both lesions in the liver and in the kidney grafts were metastases that slowly developed in the recipients over several months before being diagnosed.

The absolute risk of undiagnosed cancer transmission with solid organ transplantation is low but real. It has even been reported that in the actual organ shortage era some donors with a past history of (cured) cancer could be exceptionally considered for donation. ${ }^{11}$ This policy remains highly controversial, especially with the growing number of reported cases of cancer transmission with organ transplantation. Some cancers undiagnosed before organ donation may be discovered during multiorgan harvesting, and this discovery should interrupt the procurement. However most undiagnosed cancers are infraclinical, and there is no way to be absolutely sure that a specific donor has no malignancy. Donor autopsy has been proposed, ${ }^{1}$ but it is rarely performed and may not avoid missing some small malignant lesions. Due to the increased incidence of cancer with age, this risk should be increased in older donors, who currently are more often proposed for donation. To the authors' view, there is no reason why NHBDs should be particularly at risk of cancer transmission if the procurement is performed according to the same rules of careful inspection of the abdominal and thoracic organs. In the case described in this paper, the procurement was performed by an experienced certified transplant surgeon, and the authors' group is very much aware of this potential problem, as some cases of cancer transmission have already been encountered in the authors' experience.

Management of organ recipient with transplanted donor cancer is difficult, as cancers might develop rapidly and aggressively in immunosuppressed patients. ${ }^{12}$ This fact was at first explained by the so-called "immune surveillance hypothesis" that suggested that the immune system controls cancer development. However, the defective immune surveillance theory may not explain all the features of cancer behavior and development in immunosuppressed patients. It has recently been demonstrated that calcineurin inhibitors may directly influence cancerous cells and may induce tumor progression by a cell-autonomous mechanism that may be linked to the production of transforming growth factor- $\beta$ (TGF- $\beta$ ). ${ }^{13,14}$ On the contrary, it was proposed that mTOR inhibitors and mycophenolate mofetil-based immunosuppression might be beneficial in organ recipients with cancer. ${ }^{14}$ These data have to be further demonstrated by larger series.

Once a transmitted cancer is diagnosed in a kidney graft recipient, the graft should be removed with initiation of dialysis, and immunosuppression should be interrupted. This management, in some cases with specific chemotherapy, was successful in the majority of published cases of cancer transmission with kidney transplantation. ${ }^{1}$ For liver transplant recipients, the situation is more complicated and management may depend on the delay between donor cancer diagnosis and liver transplantation. If the cancer is diagnosed in the donor shortly after graft reperfusion, emergency retransplantation has been proposed. ${ }^{15}$ However, this policy does not allow immunosuppression interruption, and the recipient may develop generalized cancer if malignant cells have already left the graft at the time of retransplantation. ${ }^{5}$ Reducing immunosuppression and switching to a calcineurin inhibitor-free regimen, in addition to radical surgery and/or adjuvant systemic chemotherapy in selected cases, could be justified according to the tumor type and to the patient characteristics. In cases in which the donor cancer was diagnosed several months after transplantation, complete interruption of immunosuppression was proposed to induce cancer rejection. ${ }^{16}$ This policy, in addition to systemic chemotherapy and retransplantation as the rejection process induces liver graft failure, was successfully reported. ${ }^{16}$ However, cases of successful liver retransplantation are rare, ${ }^{17}$ and the management of liver recipients diagnosed with transplanted malignant tumor remains highly challenging.

\section{References}

1. Detry O, Detroz B, D’Silva M, Pirenne J, Defraigne JO, Meurisse $\mathrm{M}$, et al. Misdiagnosed malignancy in transplanted organs. Transpl Int 1993;6:50-54.

2. Penn I. Transmission of cancer from organ donors. Ann Transplant 1997;2:7-12.

3. Kauffman HM, McBride MA, Cherikh WS, Spain PC, Delmonico FL. Transplant tumor registry: donors with central nervous system tumors. Transplantation 2002;73:579-582.

4. Detry O, Honore P, Hans MF, Delbouille MH, Jacquet N, Meurisse M. Organ donors with primary central nervous system tumor. Transplantation 2000;70:244-248.

5. Lipshutz GS, Baxter-Lowe LA, Nguyen T, Jones KD, Ascher NL, Feng S. Death from donor-transmitted malignancy despite emergency liver retransplantation. Liver Transpl 2003;9:11021107.

6. Abt PL, Desai NM, Crawford MD, Forman LM, Markmann JW, Olthoff KM, et al. Survival following liver transplantation from non-heart-beating donors. Ann Surg 2004;239:87-92.

7. Wilson RE, Hager EB, Hampers CL, Corson JM, Merrill JP, 
Murray JE. Immunologic rejection of human cancer transplanted with a renal allograft. N Engl J Med 1968;278:479-483.

8. Penn I. Malignancy in transplanted organs. Transpl Int 1993;6: 1-3.

9. Kakar S, Burgart LJ, Charlton MR, Saito Y, Halling K, Thibodeau SN. Origin of adenocarcinoma in a transplanted liver determined by microsatellite analysis. Hum Pathol 2002;33: 435-436.

10. MacKie RM, Reid R, Junor B. Fatal melanoma transferred in a donated kidney 16 years after melanoma surgery. $\mathrm{N}$ Engl J Med 2003;348:567-568.

11. Kauffman HM, McBride MA, Delmonico FL. First report of the United Network for Organ Sharing Transplant Tumor Registry: donors with a history of cancer. Transplantation 2000;70:17471751.

12. Detry O, Honore P, Meurisse M, Jacquet N. Cancer in transplant recipients. Transplant Proc 2000;32:127.

13. Hojo M, Morimoto T, Maluccio M, Asano T, Morimoto K,
Lagman M, et al. Cyclosporine induces cancer progression by a cell-autonomous mechanism. Nature 1999;397:530-534.

14. Guba M, Graeb C, Jauch KW, Geissler EK. Pro- and anti-cancer effects of immunosuppressive agents used for organ transplantation. Transplantation 2004;77:1777-1782.

15. Detry O, Honore P, Jacquet N, Meurisse M. Management of recipients of hepatic allografts harvested from donors with malignancy diagnosed shortly after transplantation. Clin Transplant 1998;12:579-581.

16. Florman S, Bowne W, Kim-Schluger L, Sung MW, Huang R, Fotino $\mathrm{M}$, et al. Unresectable squamous cell carcinoma of donor origin treated with immunosuppression withdrawal and liver retransplantation. Am J Transplant 2003;4:278-282.

17. Donovan JA, Simmons FA, Esrason KT, Jamehdor M, Busuttil RW, Novak JM, Grody WW. Donor origin of a posttransplant liver allograft malignancy identified by fluorescence in situ hybridation for the Y chromosome and DNA genotyping. Transplantation 1997;63:80-84. 